\title{
Verbbedeutung und Situationsperspektivierung
}

\author{
Angelika Storrer
}

\section{Einleitung}

Dieselbe Situation kann von verschiedenen Sprechern oder von demselben Sprecher in verschiedenen Kommunikationssituationen unterschiedlich versprachlicht werden. Dabei werfen die alternativen Versprachlichungen unterschiedliche Perspektiven auf die beschriebene Situation und sind im Hinblick auf das mit der Kommunikation beabsichtigte Ziel mehr oder weniger adäquat. Während die Kriterien für kommunikative Adäquatheit sprachunabhängig formulierbar sind, wenngleich sie auch kulturspezifischen Einflüßen unterliegen, variieren die Mittel, die für die Perspektivierung von Situationen in sprachlichen Situationsbeschreibungen zur Verfügung stehen, in Abhängigkeit von einzelsprachspezifischen Parametern.

Der vorliegende Artikel befaßt sich mit der Beschreibung des deutschen Verbwortschatzes im Hinblick auf die Perspektivierungsmöglichkeiten, die mit verbalen Ausdrücken verbunden sind. Im Unterschied zu den meisten Nomina und Adjektiven eröffnen verbale Ausdrücke grundsätzlich Leerstellen, Valenzstellen oder Verbargumente genannt, mit denen die an der Situation beteiligten Entitäten in bestimmter Weise syntaktisch angeschlossen werden können. Verbale Ausdrücke, die sich prinzipiell zur Bezeichnung derselben Situation eignen, unterscheiden sich nun genau in der Anzahl ihrer Verbargumente und der Art des Beitrags der Argumente zur Verbbedeutung voneinander. Weiterhin gibt es im Deutschen wie in fast allen Sprachen systematische Perspektivierungszusammenhänge zwischen syntaktischen Konstruktionen, die mit demselben Verb oder derivationell verwandten Verben gebildet werden können. Im Vordergrund der folgenden Ausführungen steht die Frage, wie diese Perspektivierungsalternativen in einer onomasiologisch orientierten und auf Situationstypen bezogenen Beschreibung verbaler Ausdrücke berücksichtigt werden können.

Im folgenden Abschnitt werde ich zunächst die Termini "Situation", "Situationstyp" und "Situationsperspektivierung" erläutern. Diese sind Voraussetzung für das Verständnis des in Abschnitt 3 dargestellten Modells, das in der Sprachgenerierungsrichtung das Zusammenwirken der Parameter beschreibt, die die kommunikativ adäquate Auswahl zwischen verschiedenen Verbalisierungsalternativen relativ zu einer bestimmten Kommunikationssituation steuern. Die zur 
Exemplifizierung des Modells angegebenen Beispiele werden dann in Abschnitt 4 um die Beschreibung weiterer systematischer sprachlicher Mittel ergänzt, mit denen im Deutschen die Perspektivierung einer zu versprachlichenden Situation verändert werden kann. In Anbetracht der Tatsache, daß die mit einer bestimmten Verbwahl verbundenen Perspektiven weder in allgemeinen ein- und mehrsprachigen Wörterbüchern noch in Spezialwörterbüchern zur Verbvalenz systematisch erfaßt sind, folgen im abschließenden Abschnitt 5 Überlegungen dazu, wie ein Lexikon organisiert sein muß, das adäquat und redundanzfrei die sprachlichen Mittel zur Situationsperspektivierung im Deutschen berücksichtigt.

\section{Situationen, Situationstypen und Situationsperspektivierung}

Unter Situation verstehe ich ganz allgemein eine Konstellation von Entitäten mit bestimmten Eigenschaften, die an einem bestimmten Ort und zu einer bestimmten Zeit miteinander in Beziehung stehen. Situationstypen sind kognitive Schemata, in denen das Wissen eines Sprachteilnehmers über Klassen von Situationen repräsentiert ist. Diese Schemata lassen sich mit Hilfe von Datenstrukturen wie Frames und Skripts (zum Beispiel Minsky, 1980; Reimer, 1987; Schank \& Abelson, 1977) modellieren, die in der sogenannten Künstlichen Intelligenz als hierarchisch gegliederte Wissensbausteine Verwendung finden. Diese Datenstrukturen charakterisieren den für den Situationstyp typischen Ereignisverlauf in abstrakter Form und verfügen über Leerstellen, die die am Situationstyp beteiligten Entitäten spezifizieren und im vorliegenden Modell als $\mathrm{Si}$ tuationsrollen eines Situationstyps bezeichnet werden.

Situationen und die darin involvierten Entitäten und Ereignisverläufe können durch sprachliche Äußerungen beschrieben, diskutiert und erfragt werden. Wichtig für das in Abschnitt 3 dargestellte Modell ist die Unterscheidung zwischen der Äußerungssituation, der Situation, in der sich der Sprecher zum Zeitpunkt einer sprachlichen Äußerung befindet, und der Rekurssituation, der Situation, auf die in der sprachlichen Äußerung rekurriert wird. Dabei nimmt der Sprecher sowohl Äußerungssituation als auch Rekurssituation auf der Folie seines Wissens über Situationstypen wahr. Die kognitiven Repräsentationen dieser Situationen können also als Instanzen genereller Situationstyp-Frames beschrieben werden, in denen Leerstellen, Verlaufsvariablen und andere situationsspezifische Parameter mit konkreten Werten aufgefüllt sind.

Wie diese instantiierten Situationstyp-Frames nun in einer konkreten Äußerungssituation versprachlicht werden können, ist abhängig von konkreten Parametern der Äußerungssituation und von den sprachlichen Mitteln, die in einer Einzelsprache zur Verfügung stehen. Das Zusammenspiel der ausschlaggebenden Parameter wird in dem in Abschnitt 3 vorgestellten Modell skizziert. Für die Auswahl zwischen verschiedenen Verbalisierungsalternativen greife ich darin auf den Begriff der Perspektive zurück, wie ihn Fillmore (1977) im Rahmen 
seiner kasusgrammatisch orientierten Scenes- und Frames-Semantik eingeführt hat. Fillmore bindet Perspektiven an individuelle 'Szenen' (= scenes), wenngleich eine Unterscheidung zwischen individuellen Szenen und generischen Rahmen, denen sich individuelle Szenen zuordnen lassen, angestrebt wird (vergleiche die Unterscheidung in prototypical scenes, real-world-scenes und schematic scenes bei Fillmore (1977, S. 70). Im Gegensatz dazu bezieht der vorliegende Ansatz den Begriff der Perspektive auf Situationstypen und deren Versprachlichungsalternativen. Auf diese generischen Situationstypen und Situationsrollen können sprachlich induzierte Perspektivenunterschiede, die sich zum Beispiel über grammatische Diathesen, Intransitivierung oder Wortbildungsmittel ergeben, systematisch bezogen werden. Weiterhin unterscheide ich zwischen den Termini "Perspektive" und "Perspektivierung": Ersterer bezieht sich auf die Interpretation einer Äußerung beim Sprachverstehensprozeß, letzterer bezieht sich auf die Verbalisierung einer Situation im Sprachgenerierungsprozeß.

Unter der Perspektivierung eines Situationstyps verstehe ich eine Auswahl an Informationen zum Situationstyp, die für eine sprachliche Äußerung getroffen wurde. Dabei unterscheide ich zwei Arten von Perspektivierung:

1. Mit Rollenperspektivienung bezeichne ich die Auswahl von Art und Anzahl der am Situationstyp beteiligten Rollen. Mit verschiedenen verbalen Ausdrücken kann die in der Situation gegebene Instantiierung dieser Rollen in verschiedener Weise versprachlicht oder offengelassen werden.

2. Mit Verlaufsperspektivierung bezeichne ich die Auswahl zusätzlicher Aspekte, durch die der im Situationstyp standardmäßig erfaßte Situationsablauf modifiziert oder in spezifischer Weise ausgeleuchtet wird.

Mit verschiedenen verbalen Ausdrücken einer Einzelsprache können verschiedene Perspektiven auf denselben Situationstyp geworfen werden. Die Gesamtheit der Perspektivierungsmöglichkeiten eines verbalen Ausdrucks bezeichne ich als dessen Perspektivierungspotential. In einer konkreten Äußerung kann dieses Potential in der einen oder anderen Weise ausgeschöpft werden. Weiterhin kann in einer konkreten Äußerung die gewählte Perspektivierung um äußerungsbezogene Parameter akzentuiert und verfeinert werden, wie die in der Äußerung gewählte Informationsstruktur und die intonatorische oder topologische Fokussierung bestimmter Äußerungseinheiten. Diese Parameter werden im vorliegenden Ansatz nicht berücksichtigt.

\section{Situationsperspektivierung in einem Sprachgenerierungsmodell}

Um die Perspektivierungsmöglichkeiten einer Einzelsprache systematisch beschreiben zu können, bedarf es eines Modells, das alle hierfür relevanten Parameter erfaßt. In der Sprachanalyserichtung kann dann formal spezifiziert werden, wie die Parameter die korrekte Interpretation sprachlicher Äußerungen in 
einer weltbezogenen Strukturbeschreibung ermöglichen. In der Sprachgenerierungsrichtung geht es darum, das Zusammenwirken der Parameter zu bestimmen, die in einer Kommunikationssituation die Auswahl einer geeigneten Verbalisierung für eine kommunikativ angemessene Äußerung steuern. Ein solches Modell für die Generierungsrichtung wird im folgenden Abschnitt vorgestellt werden. Es handelt sich dabei um eine im Hinblick auf die Perspektivierungsmöglichkeiten modifizierte und erweiterte Fassung des Modells der Situationsvalenz (Storrer, 1992). Semantisch lehnt sich das Modell an die Kommunikantensemantik (Mudersbach, 1984) an, die Repräsentation von sprach- und weltbezogenen Strukturen orientiert sich, wie im vorherigen Abschnitt erläutert, an den in der sogenannten Künstlichen Intelligenz und der Kognitionswissenschaft eingeführten Frame- und Skript-Konzepten.

Ausgangspunkt des Modells ist eine Äußerungssituation AS, in der ein Sprecher S mit einem Hörer beziehungsweise einem Kollektiv von Hörern $\mathrm{H}$ kommuniziert. Der Sprecher verfügt zu diesem Zeitpunkt über einen bestimmten Informationsstand INF/S und hat Hypothesen über den Informationsstand des Hörers INF/H/S. S will dabei $\mathrm{H}$ zu einer bestimmten Situation, der Rekurssituation RS, etwas Neues und Relevantes mitteilen und konzipiert dazu eine entsprechende Äußerung U. Zum Zeitpunkt $t$, in dem $S$ diese Äußerung konzipiert, kreist die Kommunikation um einen bestimmten Aufmerksamkeitsbereich AM. Außerdem verfolgt der Sprecher mit seiner Äußerung eine bestimmte Absicht, die von seiner Interessenslage INT/S zum Äußerungszeitpunkt geleitet ist.

Die Äußerungssituation AS sowie das Sprach- und Weltwissen des Sprechers GS/S und seine Hypothesen vom Sprach- und Weltwissen des Hörers GS/H/S sind parametrisiert. Einige der Parameter beziehen sich auf Wissensbestände, die dem Sprecher aufgrund seiner Zugehörigkeit zu sprachlichen und kulturellen Gemeinschaften und seiner individuellen Biographie zur Verfügung stehen. Hierzu gehören das Wissen über standardmäßige Situationsabläufe, modelliert mittels Situationstypen, und über deren charakteristische Beteiligte, modelliert als Situationsrollen. Andere Parameter beziehen sich auf die Äußerungssituation selbst: Hierzu zählen Zeitpunkt und Ort der Äußerung t-p/U, der momentane Aufmerksamkeitsbereich AM sowie die Interessenslage des Sprechers INT/S. Außerdem hat S zum Zeitpunkt der Äußerung die Rekurssituation RS, die ja in U auf bestimmte Weise versprachlicht werden soll, einem bestimmten Situationstyp Sit-Typ(RS) zugeordnet.

Das Modell operiert nun in zwei Schritten über diesen Parametern:

1. Im Konzeptualisierungsschritt werden die Rollenperspektivierung, die Verlaufsperspektivierung und die kommunikative Perspektivierung festgelegt.

2. Im Verbalisierungsschritt wird entschieden, mit welchen verbalen Ausdrücken die gewählte Perspektivierung kommunikativ angemessen verbalisiert werden kann. 


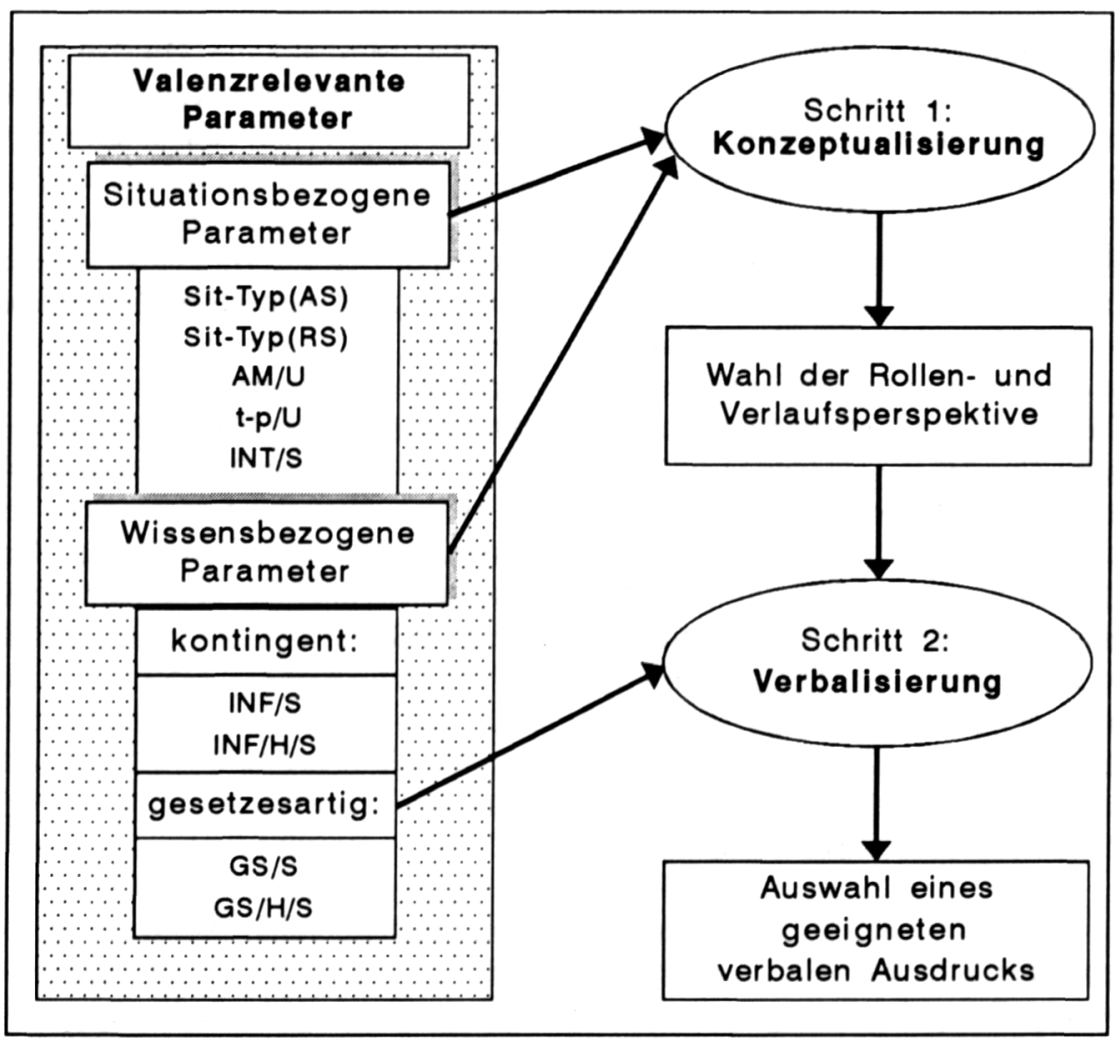

Abbildung 1: Ein Modell zur Situationsperspektivierung.

Der Zusammenhang zwischen den Komponenten wird in Abbildung 1 graphisch verdeutlicht. Während im ersten Schritt vor allem situationsspezifische Parameter der Äußerungssituation eine Rolle spielen, operiert der zweite Schritt über sprachbezogenen Parametern, die sich auf das Perspektivierungspotential verbaler Ausdrücke beziehen. Der erste Schritt kann entfallen, wenn, wie bei der Übersetzung von Einzelsprachen, bereits durch die Ausgangssprache eine bestimmte Konzeptualisierung vorliegt, für die nun eine angemessene Versprachlichung in der Zielsprache gesucht werden muß.

\subsection{Der Konzeptualisienungsschritt}

Die für den Konzeptualisierungsschritt relevanten Parameter und ihr Zusammenspiel werden hier nur informell skizziert werden (Details finden sich in Storrer, 1992). Der Begriff der kommunikativen Angemessenheit wurde dort an die von Grice (1975) postulierten Konversationsmaximen angebunden, wodurch die Wahl der Rollenperspektivierung relativ zu den Parametern der Äuße- 
rungssituation durch allgemeine Prinzipien erklärt werden kann. Hierbei spielt zunächst der Informationsstand des Sprechers INF/S zum Äußerungszeitpunkt eine Rolle: Situationsrollen, deren Belegung dem Sprecher zum Äußerungszeitpunkt unbekannt sind, können in U höchstens erfragt oder als Indefinitpronomen realisiert, nicht aber als Komplement spezifiziert werden. Ein weiterer Faktor sind die Hypothesen, die der Sprecher über den Informationsstand des Hörers INF/H/S hat: Situationsrollen, deren Belegung dem Hörer bereits bekannt sind, werden im Normalfall nur thematisch, das heißt in anknüpfender Wiederaufnahme von bereits Gesagtem, nicht aber rhematisch, das heißt als Mitteilung neuer Information, verwendet. Dasselbe gilt für Rollenbelegungen, die für den Hörer aus der Sicht des Sprechers aus der aktuellen Äußerungssituation AS beziehungsweise dem Wissen des Hörers über den Situationstyp der Äußerungssituation Sit-Typ(AS) beziehungsweise dem Situationstyp der Rekurssituation Sit-Typ(RS) erschließbar sein sollten. Schließlich kann eine Situationsrolle schlichtweg deshalb nicht verbalisiert werden, weil es der momentanen Interessenslage des Sprechers INT/S abträglich ist, diese Information an den Hörer weiterzugeben.

\subsection{Der Verbalisierungsschritt}

Im Verbalisierungsschritt geht es nun darum, einen verbalen Ausdruck in der gegebenen Einzelsprache zu finden, mit dem die im Konzeptualisierungsschritt gewählte Perspektivierung optimal versprachlicht werden kann. In bezug auf die Rollenperspektivierung läßt sich die Auswahl zwischen Verbalisierungsalternativen modellieren als zweifacher Musterabgleich zwischen dem als FrameStruktur dargestellten Situationstyp der Rekurssituation Sit-Typ(RS) und den Valenzrahmen der verbalen Ausdrücke, die prinzipiell zur Bezeichnung des Rekurssituationstyps geeignet sind.

Die Grundidee dieses Verfahrens möchte ich im folgenden anhand der Verbalisierungsalternativen für den Situationstyp "Lüge" skizzieren. Ich stütze mich dabei einerseits auf Beschreibungen aus der ESKA-Datenbank, die im Rahmen des Projekts Erklärende Synonymik kommunikativer Ausdrücke (ESKA) aufgebaut wird (vergleiche Harras \& Winkler, 1994), andererseits auf Verwendungsbeispiele aus den Textkorpora des Instituts für deutsche Sprache.

Das semantische Beschreibungskonzept in ESKA geht aus von einem allgemeinen Rekurssituationstyp Kommunikative Handlung mit den standardmäßigen Situationsrollen des Sprechers, des Hörers (der Adressat der kommunikativen Handlung), des Äußerungsproduktes und einer komplexen kommunikativen Einstellung des Sprechers. Speziellere Rekurssituationstypen ergeben sich durch die Spezifizierung der Situationsrollen mit typischen Merkmalen; die Beschreibung kommunikativer Ausdrücke einer Einzelsprache beziehungsweise der Kontrastierung verschiedener Einzelsprachen wird auf diese spezielleren 
Rekurssituationstypen bezogen (vergleiche Harras [1995] sowie Winkler in diesem Band).

Der spezielle Rekurssituationstyp "Lüge" gehört zu der Gruppe der Repräsentative, das heißt der Behauptens- und Mitteilensprädikate; seine Verwendungsbedingungen lassen sich vereinfachend folgendermaßen formulieren (für eine detailliertere Beschreibung verweise ich auf Harras [1995] und die Einträge in der ESKA-Datenbank):

(a) Der Sprecher S hält die in U versprachlichte Proposition $\mathrm{P}$ nicht für wahr.

(b) Der Sprecher $\mathrm{S}$ will, daß der Hörer $\mathrm{H}$ die in $\mathrm{U}$ versprachlichte Proposition $\mathbf{P}$ für wahr hält.

Diese Rekurssituation kann nun Kommunikationsgegenstand einer sprachlichen Äußerung in einer Äußerungssituation sein, an der ebenfalls ein Sprecher einem Hörer gegenüber eine sprachliche Äußerung hervorbringt. Da in dieser Konstellation sowohl Äußerungs- als auch Rekurssituation zum generellen Situationstyp "Kommunikative Handlung" gehören, müssen die Situationsrollen des Sprechers und des Hörers und des Äußerungsprodukts entsprechend als ASSprecher versus RS-Sprecher, AS-Hörer versus RS-Hörer etc. indiziert werden, wobei AS die Äußerungssituation und RS die Rekurssituation bezeichnet.

Die Rekurssituation mit den Rollen RS-Sprecher, RS-Hörer und RS-Proposition ist nun, wie in Abbildung 2 graphisch veranschaulicht ist, in den Situationstyp der Äußerungssituation eingebettet. In dieser möchte der AS-Sprecher einem AS-Hörer etwas Neues und Relevantes zu einer Rekurssituation vom Typ "Lüge" mitteilen. Im vorangegangenen Konzeptualisierungsschritt hat er dazu eine bestimmte Anzahl von Situationsrollen ausgewählt und sich entschieden, welche Aspekte des Situationsverlaufs er besonders akzentuieren möchte, das heißt, Rollen- und Verlaufsperspektive sind Parameter der im ersten Schritt erfolgten Konzeptualisierung. Um diese Konzeptualisierung adäquat verbalisieren zu können, muß nun geprüft werden, welche verbalen Ausdrücke, mit denen der Rekurssituationstyp "Lüge" prinzipiell beschrieben werden kann, genau diese Rollen- und Verlaufsperspektive realisieren können. In bezug auf die Rollenperspektive sind das genau die verbalen Ausdrücke, die

(a) alle gewählten Situationsrollen als syntaktische Komplemente anschlieBen können;

(b) keine nicht gewählten Situationsrollen syntaktisch anschließen müssen. 


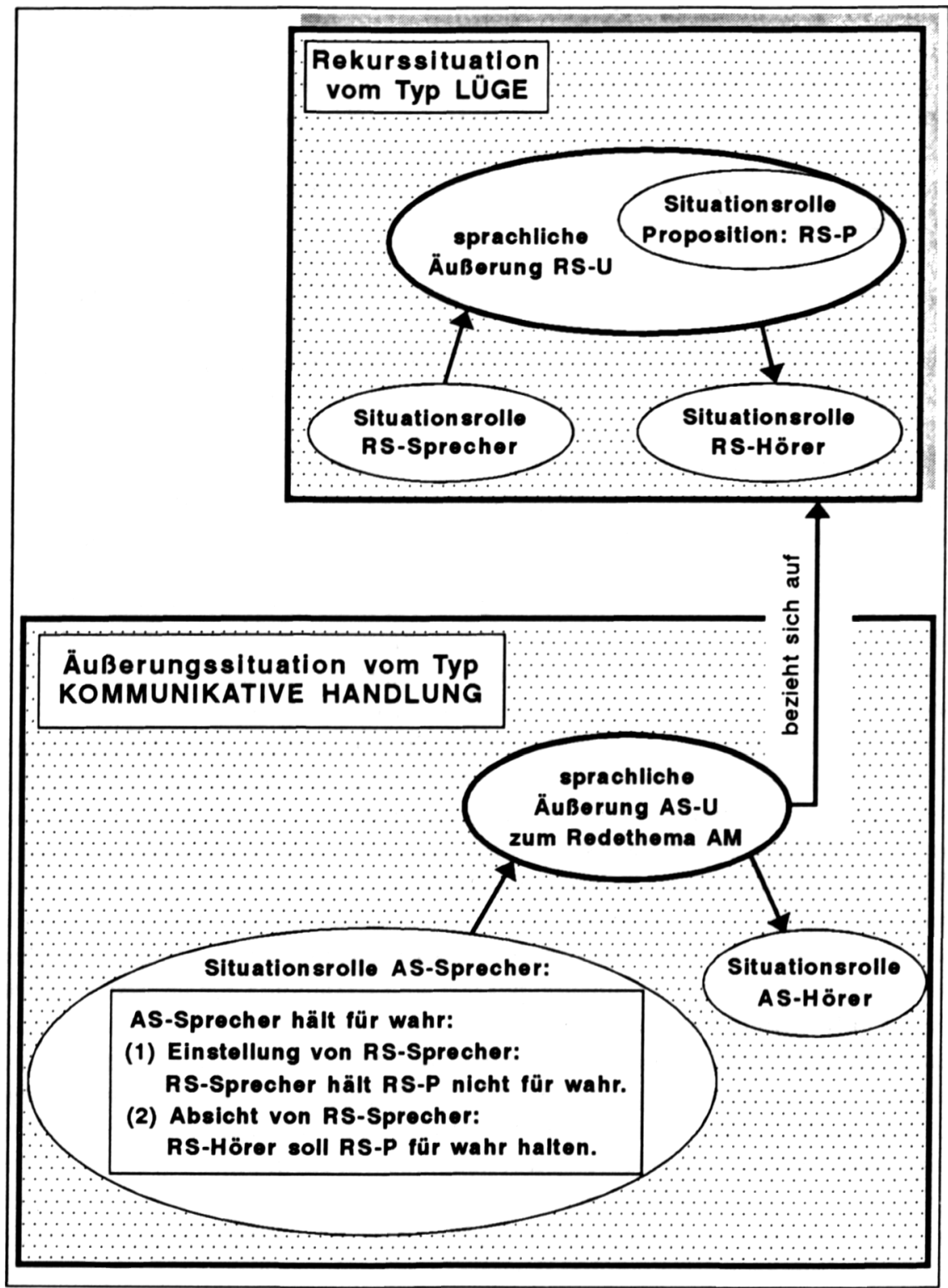

Abbildung 2: Die in eine Äußerungssituation eingebettete Rekurssituation vom Typ "Lüge". Die rollenbezogene Perspektivierung kann mit den Verben "lügen", "belügen", "anlügen", "erlügen" oder "vorlügen", die verlaufsbezogene Perspektivierung mit den Verben "rumlügen", "sich durchlügen" oder "zusammenlügen" ausgedrückt werden (siehe Text). 
Im Deutschen gibt es eine Reihe verbaler Ausdrücke, die für die Beschreibung dieses Situationstyps prinzipiell geeignet sind. Dazu gehören das Verb "lügen" und die durch Wortbildungssmittel abgeleiteten Verben "belügen", "anlügen", "vorlügen", "erlügen", "rumlügen", "sich durchlügen" und "zusammenlügen" (die Verben "schwindeln", "flunkern" und ihre Derivate, die ebenfalls zu diesem Wortfeld gehören, bleiben hier unberücksichtigt, da sie sich nicht auf die Perspektivierung, sondern auf die Bewertung der Lüge durch den AS-Sprecher beziehen; vergleiche Harras, 1995, S. 110f.). Diese verbalen Ausdrücke des Lügens unterscheiden sich in bezug auf das bereitgestellte Rollenpotential erheblich (siehe Tabelle 1):

Mit dem einfachen Verb "lügen" wird vor allem der RS-Sprecher (Lügner) perspektiviert. Darüber hinaus fanden sich in den Mannheimer Korpora einige Korpusbelege für den Anschluß der RS-Proposition (der Lüge) in direkter Rede, wie in Beispielsatz (2).

Die angegebenen Beispiele sind der besseren Verständlichkeit wegen keine authentischen Korpusbelege, sondern selbstkonstruierte Beispielsätze; gegebenenfalls werden diese als ungrammatisch $\left(^{*}\right)$ beziehungsweise als nur bedingt grammatisch (?) bewertet.

(1) Peter log.

(2) "Die Hausaufgaben sind schon fertig", log Peter.

(3) ? Peter log gegenüber der Mutter.

Für den Anschluß der Rolle des RS-Hörers (des Belogenen) in der Art von (3) fand sich kein Beleg. Ein kompetenter AS-Sprecher wird bei der Rollenkonstellation RS-Sprecher und RS-Hörer auf die Verben "belügen" und "anlügen" ausweichen, die beide die Rolle des Belogenen nicht nur versprachlichen können (vergleiche (4) und (5)), sondern sogar müssen (vergleiche (9)):

(4) Peter belügt die Mutter.

(5) Peter lügt die Mutter an.

(6) Die Mutter wurde belogen/angelogen.

(7) ? "Die Hausaufgaben sind schon fertig", belog Peter die Mutter.

(8) "Die Hausaufgaben sind schon fertig", log Peter die Mutter an.

(9) * Peter belügt/lügt an.

Für den Anschluß von RS-U in der Art von (7) und (8) fand sich kein Beleg. "Anlügen" erscheint mir in dieser Konstruktion jedoch besser geeignet als "belügen". Das geeignetste Perspektivierungspotential zur Realisierung der in (7) und (8) angezeigten Rollenkonstellation (RS-Sprecher, RS-Hörer, RS-Proposition) besitzt ohnehin das Verb "vorlügen":

(10) Peter log der Mutter vor, die Hausaufgaben seien schon fertig.

(11) "Die Hausaufgaben sind schon fertig", $\log$ Peter der Mutter vor. 
(12) * Peter log der Mutter vor.

(13) * "Die Hausaufgaben sind schon fertig", log Peter vor.

(14) Der Mutter wurde vorgelogen, die Hausaufgaben seien schon fertig.

Tabelle 1: Das Rollenpotential der Verben des Lügens im Deutschen.

\begin{tabular}{|c|c|c|c|}
\hline Verb & RS-Sprecher & RS-Hörer & RS-Proposition \\
\hline lügen & $\begin{array}{l}\text { Nominativ- } \\
\text { komplement }\end{array}$ & $\begin{array}{l}\text { Adjunkt } \\
\text { (gegenüber } x \text { ) }\end{array}$ & $\begin{array}{l}\text { (direkte Rede) } \\
\text { Adjunkt } \\
\text { (als } \times \text { sagte, daB p) }\end{array}$ \\
\hline belügen & $\begin{array}{l}\text { Nominativ- } \\
\text { komplement }\end{array}$ & $\begin{array}{l}\text { Akkusativ- } \\
\text { komplement }\end{array}$ & $\begin{array}{l}\text { Adjunkt } \\
\text { (als } \times \text { sagte, daB p) }\end{array}$ \\
\hline $\begin{array}{l}\text { belügen } \\
\text { (w-Passiv) }\end{array}$ & $\begin{array}{l}\text { (von Präposi- } \\
\text { tionalphrase) }\end{array}$ & $\begin{array}{l}\text { Nominativ- } \\
\text { komplement }\end{array}$ & \\
\hline anlügen & $\begin{array}{l}\text { Nominativ- } \\
\text { komplement }\end{array}$ & $\begin{array}{l}\text { Akkusativ- } \\
\text { komplement }\end{array}$ & $\begin{array}{l}\text { direkte Rode } \\
\text { Adjunkt } \\
\text { (als } \times \text { sagte, daB p) }\end{array}$ \\
\hline $\begin{array}{l}\text { anlügen } \\
\text { (w-Passiv) }\end{array}$ & $\begin{array}{l}\text { (von Präposi- } \\
\text { tlonalphrase) }\end{array}$ & $\begin{array}{l}\text { Nominativ- } \\
\text { komplement }\end{array}$ & \\
\hline vorlügen & $\begin{array}{l}\text { Nominativ- } \\
\text { komplement }\end{array}$ & $\begin{array}{l}\text { Dativ- } \\
\text { komplement }\end{array}$ & $\begin{array}{l}\text { indirekte Rede } \\
\text { Akkusativ- } \\
\text { komplement }\end{array}$ \\
\hline $\begin{array}{l}\text { vorlügen } \\
\text { (w-Passiv) }\end{array}$ & $\begin{array}{l}\text { (von Präposi- } \\
\text { tionalphrase) }\end{array}$ & $\begin{array}{l}\text { Dativ- } \\
\text { komplement }\end{array}$ & $\begin{array}{l}\text { indirekte Rede } \\
\text { Akkusativ- } \\
\text { komplement }\end{array}$ \\
\hline erlügen & $\begin{array}{l}\text { Nominativ- } \\
\text { komplement }\end{array}$ & & $\begin{array}{l}\text { Akkusativ- } \\
\text { komplement }\end{array}$ \\
\hline $\begin{array}{l}\text { erlügen } \\
\text { (s-Passiv) }\end{array}$ & & & $\begin{array}{l}\text { Akkusativ- } \\
\text { komplement } \\
\text { daB-Satz }\end{array}$ \\
\hline rumlügen & $\begin{array}{l}\text { Nominativ- } \\
\text { komplement }\end{array}$ & & \\
\hline $\begin{array}{l}\text { sich } \\
\text { durchlügen }\end{array}$ & $\begin{array}{l}\text { Nominativ- } \\
\text { komplement }\end{array}$ & & \\
\hline $\begin{array}{l}\text { zusammen- } \\
\text { lügen }\end{array}$ & $\begin{array}{l}\text { Nominativ- } \\
\text { komplement }\end{array}$ & $\begin{array}{l}\text { Adjunkt } \\
\text { (gegenüber } x \text { ) }\end{array}$ & $\begin{array}{l}\text { Indefinit- } \\
\text { pronomen }\end{array}$ \\
\hline
\end{tabular}

Bei diesem Verb sind jedoch, wie (12) und (13) zeigen, alle drei Komplemente syntaktisch obligatorisch, das heißt, es kann in einer Aktivkonstruktion nur gewählt werden, wenn wirklich alle drei Situationsrollen versprachlicht werden 
sollen. Weglaßbar ist lediglich die Rolle des RS-Sprechers in einer Passivkonstruktion wie (14).

Nimmt man die passivischen Konstruktionen (6) und (14) hinzu, können mit den Verben "lügen", "belügen"/"anlügen" und "vorlügen" folgende Rollenkonstellationen versprachlicht werden:

- $\quad$ RS-Sprecher \& RS-Hörer \& RS-Proposition ("vorlügen");

- $\quad$ RS-Sprecher \& RS-Proposition ("lügen");

- $\quad$ RS-Sprecher \& RS-Hörer ("belügen", "anlügen" im Aktiv);

- $\quad$ RS-Hörer \& RS-Proposition ("vorlügen" im Passiv);

- $\quad$ RS-Sprecher ("lügen");

- $\quad$ RS-Hörer ("belügen", "anlügen" im Passiv).

Zum Ausdruck der Rolle der RS-Proposition dient das Zustandspassiv des Verbs "erlügen" wie in Beispiel (15):

(15) Die Geschichte ist erlogen.

(16) Peter erlügt eine Geschichte.

(17) * Peter erlügt der Mutter eine Geschichte.

(18) Peter hat sich einen Kredit erlogen.

Nach den vorliegenden Korpusanalysen kommt "erlügen" vornehmlich im $\mathrm{Zu}$ standspassiv vor, was damit erklärt werden kann, daß dies die präferierte Möglichkeit ist, mit den angegebenen Verben die RS-Proposition alleine zu perspektivieren. Prädikativkonstruktionen (zum Beispiel "ist nicht wahr", "stimmt nicht") beziehen sich nicht direkt auf die kommunikative Handlung und sind somit keine echten Alternativen. Statt des einfachen Partizips kann auch die Zwillingskonstruktion "erstunken und erlogen" gewählt werden, womit der ASSprecher Empörung über die RS-Lüge zum Ausdruck bringt. Die aktivische Konstruktion kann mit einer Verlaufsperspektivierung verbunden sein, wie sie für die Verbalpartikel "er-" typisch ist: Die vom Basisverb bezeichnete Handlung führt zum Erwerb eines im Akkusativkomplement ausgedrückten Besitzes ("erarbeiten", "erheiraten", etc.; vergleiche Stiebels, 1994, S. 110ff.). Typisch für diese Verlaufsperspektivierung ist das Vorhandensein eines Reflexivpronomens wie in (18), daneben existieren aber auch reguläre Varianten mit effiziertem Akkusativkomplement und ohne possessive Implikationen wie in (16). Nicht anschließbar ist die Rolle des RS-Hörers (vergleiche (17)).

Primär verlaufsbezogen ist die Perspektivierung, die mit den Verben "rumlügen", "zusammenlügen" und "sich durchlügen" eingebracht wird. Die mit diesen Verben eröffneten Möglichkeiten der Rollenperspektivierung lassen sich auf die Verlaufsperspektive zurückführen, die mit dem Präfix eingebracht wird, und sind somit sekundär: Das Präfix "zusammen-" vermittelt die Perspektive einer akkumulierenden Wiederholung der im Simplexverb ausgedrückten Tätigkeit, das heißt im Falle von "zusammenlügen" der Aufbau eines Lügengebäudes 
aus mehreren Lügen. Es verwundert deshalb nicht, daß mit diesem Verb die RS-Rolle nicht als direkte Rede realisiert werden kann (vergleiche (21)). Allerdings darf diese Rolle auch nicht einfach weggelassen werden (vergleiche (20)), sie muß vielmehr durch das Indefinitpronomen "etwas" (umgangssprachlich "was"; vergleiche (19)) realisiert sein.

(19) Peter lügt ganz schön was zusammen.

(20) * Peter lügt zusammen.

(21) * "Die Hausaufgaben sind fertig", log Peter zusammen.

(22) Er log seinen Eltern gegenüber ganz schön etwas zusammen.

Die Rolle des RS-Hörers kann bei "zusammenlügen", wie bei "lügen" auch, allerdings als Adjunkt angeschlossen werden (zum Beispiel "seinen Eltern gegenüber" in Satz (22)). Die Rolle ist dann allerdings schwächer perspektiviert als bei der Komplementrealisierung dieser Rolle bei "belügen", "anlügen" und "vorlügen".

Bei "sich durchlügen" können die Rollen des RS-Hörers und der RS-Proposition überhaupt nicht angeschlossen werden (vergleiche (27), (28)). Auch dies läßt sich mit der spezifischen Verlaufsperspektivierung erklären, die durch das Präfix eingebracht wird: Mit "sich durchlügen" wird vor allem der Weg hin zu einem Ziel perspektiviert. Die Rolle des Äußerungsprodukts läßt sich lediglich als mit-Präpositionalphrase wie in (25) anschließen, wodurch diese Rolle als Mittel zur Erreichung des Ziels beschrieben wird:

(23) Peter log sich durch.

(24) Peter log sich durch alle Instanzen bis zum Freispruch durch.

(25) ? Peter log sich mit seiner Geschichte bis zum Freispruch durch.

(26) * Peter log sich durch, als er sagte, er sei unschuldig.

(27) * Peter log sich gegenüber dem Richter durch.

Der wegstreckenorientierten Perspektivierung von "sich durchlügen" steht die mit dem Verb "rumlügen" verbundene Verlaufsperspektivierung entgegen: Mit dem Präfix "rum-" kann die im Simplexverb implizierte Zielgerichtetheit, Strukturiertheit oder Resultatsbezogenheit eines Zustands oder Ereignisses annulliert beziehungsweise das Nichtvorhandensein solcher Merkmale betont werden (vergleiche Blume, 1993, S. 59ff.). "Rumlügen" impliziert also im Gegensatz zu "lügen" genau eine unstrukturierte, nicht zielgerichtete Wiederholung von lügen-Handlungen. Diese Perspektive verhindert die Realisierung der Rolle des RS-Hörers und/oder einer einzelnen RS-Proposition (vergleiche (29) und (30)).

(28) Peter lügt rum.

(29) * Peter log gegenüber der Mutter rum.

(30) * "Die Hausaufgaben sind schon fertig", log Peter rum. 
Wichtig für die vorliegende Modellierung ist, daß die mit der Verbwahl verbundene Verlaufsperspektivierung grundsätzlich aus der Beobachterperspektive des AS-Sprechers vorgenommen wird. Diesem können die vom RS-Sprecher ausgeführten kommunikativen Handlungen unstrukturiert und nicht zielgerichtet erscheinen, weshalb er gegebenenfalls die Rekurssituation dem AS-Hörer gegenüber mit "rumlügen" statt mit "lügen" versprachlicht, obwohl der RS-Sprecher in der Rekurssituation durchaus Ziel und Zweck mit seinen Lügen verband.

Die Unterscheidung von Rekurs- und Äußerungssituation ist speziell für die Beschreibung der Verben der Lügen-Gruppe wichtig. Im Gegensatz zu prototypischen Verben des kommunikativen Handelns wie "mitteilen" in Satz (31) kann nämlich die mit Satz (32) intendierte kommunikative Handlung nicht erfolgreich durchgeführt werden.

(31) Ich möchte Ihnen mitteilen, daß der Solidaritätszuschlag bald wieder gesenkt werden wird.

(32) Ich möchte Ihnen vorlügen, daß der Solidaritätszuschlag bald wieder gesenkt werden wird.

Aus der Unterscheidung von Äußerungs- und Rekurssituation ergibt sich eine einfache Erklärung dieses Phänomens: Spezifisch für den Rekurssituationstyp "Lüge" ist, daß der RS-Sprecher zur Verfolgung seiner Zielsetzung (RS-Sprecher hält für wahr: P) dem RS-Hörer das Gegenteil seiner eigenen epistemischen Einstellung zu P (AS-Sprecher hält nicht für wahr: P) kundtut. In einem Satz wie (31) würde der RS-Sprecher dem RS-Hörer einerseits vermitteln, daß er die im daß-Satz ausgedrückte Proposition für wahr hält, während er andererseits durch die Verwendung der Verbs "vorlügen" im Matrixsatz zu erkennen gibt, daß er dieselbe Proposition nicht für wahr hält - eine Konstellation, mit der beim RS-Hörer kaum die beabsichtigte Wirkung (RS-Hörer soll $P$ für wahr halten) erzielt werden kann. Diese Schwierigkeit tritt nicht auf, wenn eine Situation vom Typ "Lüge" Gegenstand der Kommunikation zwischen AS-Sprecher und AS-Hörer in einer Äußerungssituation ist, wie die Gegenüberstellung von (33) und (34) zeigt:

(33) Der Politiker teilte Ihnen mit, daß der Solidaritätszuschlag bald wieder gesenkt werden wird.

(34) Der Politiker log Ihnen vor, daß der Solidaritätszuschlag bald wieder gesenkt werden wird.

In diesem Fall dienen die Verben der Lügen-Gruppe genau dazu, den Täuschungsversuch von RS-Sprecher gegenüber dem RS-Hörer in seinem Verlauf zu beleuchten und zu bewerten. Aus der Perspektive des AS-Sprechers kann also in (34) ebenso von einem Gelingen beziehungsweise Mißlingen der LügenHandlung gesprochen werden wie von einem Gelingen oder Mißlingen der Mit- 
teilen-Handlung in (33). Die Verwendung der Verben der Lügen-Gruppe in der ersten Person Präsens in (32) ist ein Spezialfall mit gesonderten Verwendungsbedingungen, die sich durch die raum-zeitliche und personale Identität von Äußerungs- und Rekurssituation ergibt. In allen anderen Formen können die Verben zum Rekurssituationstyp "Lüge" analog zu anderen kommunikativen Verben beschrieben werden.

Am Beispiel des Situationstyps "Lüge" läßt sich zeigen, daß eine außersprachliche Situation mit verschiedenen verbalen Ausdrücken in durchaus unterschiedlicher Art und Weise perspektiviert werden kann. Im folgenden Kapitel werde ich zeigen, wie Perspektivierungsunterschiede im Deutschen durch systematisch beschreibbare sprachliche Mittel erzielt werden können.

\section{Perspektivierungstypen und ihre Realisierungsmöglichkeiten im Deutschen}

Bereits an den Beispielen der im vorigen Abschnitt diskutierten lügen-Verbgruppe dürfte deutlich geworden sind, daß es keine Eins-zu-eins-Entsprechung zwischen sprachlichen Mitteln und Perspektivierungstypen gibt. Vielmehr können mit denselben sprachlichen Mitteln verschiedene Arten der Perspektivierung verbunden sein. Umgekehrt kann dieselbe Art der Perspektivierung durch verschiedene sprachliche Mittel erreicht werden. Trotzdem soll im folgenden unterschieden werden zwischen sprachlichen Mitteln, die primär der Rollenperspektivierung dienen (4.1) und solchen, die primär der Verlaufsperspektivierung dienen und nur sekundär Auswirkung auf die Rollenperspektivierung haben (4.2). Diese Trennung ist dadurch motiviert, daß sich die Unterschiede in der Rollenperspektivierung im Lexikon durch Relationierung von Verbargumenten und Situationsrollen modellieren lassen, während bei der Verlaufsperspektivierung kompositionelle Prozesse mitberücksichtigt werden müssen, die eine Veränderung der Argumentstruktur nach sich ziehen können, aber nicht müssen.

\subsection{Rollenperspektivierung im Deutschen}

Ein verbaler Ausdruck im Deutschen verfügt auf semantischer Ebene über eine bestimmte Anzahl von Leerstellen, im weiteren Verbargumente genannt, die auf syntaktischer Ebene in bestimmter Art und Weise als Komplemente realisiert werden können. Verbargumente vermitteln zwischen den Situationsrollen eines Situationstyps und deren Versprachlichungsmöglichkeiten auf syntaktischer Ebene. Verbale Ausdrücke, die sich in der Anzahl ihrer Argumente und der Art ihrer syntaktischen Realisierung unterscheiden, verfügen deshalb automatisch über ein unterschiedliches Potential zur Rollenperspektivierung. Im folgenden sollen verschiedene Fälle an prototypischen Beispielen unterschieden werden. 
(a) Typ befehlen - anordnen: Zwei verbale Ausdrücke unterscheiden sich durch die Anzahl ihrer Argumente. Ein Beispiel ist das Verbpaar "befehlen" und "anordnen":

(35) Paul befiehlt dem Kind, aufzuräumen.

(36) * Paul ordnet dem Kind an, aufzuräumen.

Man sieht, daß bei "anordnen" die Rolle des 'Befehlsempfängers', die bei "befehlen" als Dativkomplement realisierbar ist, nicht im Matrixsatz verbalisiert werden kann.

Während es sich bei diesem Beispiel um eine idiosynkratische Opposition zwischen zwei derivationell unabhängigen Verbausdrücken handelt, gibt es im Deutschen systematische Möglichkeiten zur Erhöhung oder Verminderung der Anzahl der Verbargumente. In Lexikologie und Grammatik gut erforscht sind die als Kausativierung beziehungsweise als Rezessivierung bezeichneten Argumentstrukturveränderungen. Rezessivierung liegt beispielweise vor zwischen dem kausativen Änderungsverb "erweitern" und seinem reflexiven Pendant "sich ändern": Während bei ersterem die Rolle der Verursacherin des Erweiterungsprozesses die Subjektstelle einnimmt, bleibt diese Rolle bei letzterem unausgedrückt. Die Subjektstelle wird nun durch das erweiterte Objekt belegt, während das akkusativische Reflexivpronomen semantisch leerläuft.

(37) Die neue Lehrstuhlinhaberin erweiterte das Forschungsspektrum des Instituts.

(38) Das Forschungsspektrum erweiterte sich.

$\mathrm{Da} ß$ dieses Rezessivierungsmuster typisch ist für das Verhältnis von kausativen und einfachen Änderungsverben, zeigen die ausführlichen Beschreibungen dieser Verbgruppen im semantischen Valenzwörterbuch Verben in Feldern (Schumacher, 1986). Die semantische Umkehrung der Rezessivierung, die Kausativierung, wird im Deutschen häufig durch Konstruktionen mit Funktionsverbgefügen erzeugt. Funktionsverbgefüge bestehen aus einer Präpositional- oder Nominalphrase, die durch ein Verbal- oder Adjektivabstraktum (zum Beispiel "das Heulen", "die Wut") belegt ist, und einem Funktionsverb, das relativ zu seiner Bedeutung als Vollverb semantisch stark eingeschränkt ist (vergleiche Polenz, 1987, sowie Storrer \& Schwall, 1995). Funktionsverben, mit denen eine Kausativierung des vom nominalen Teil ausgedrückten Prozesses/Zustands verbunden ist, sind beispielsweise "bringen" und "versetzen" in (40) und (42):

(39) Das Kind heult.

(40) Der Vater bringt das Kind zum Heulen.

(41) Das Kind ist wütend.

(42) Der Vater versetzt das Kind in Wut. 
Damit verbunden ist eine inchoative Verlaufsperspektivierung, die das Einsetzen eines Prozesses oder Zustands bezeichnet, eingeleitet durch den in der Subjektstelle ausgedrückten Urheber. Neben dieser systematischen Möglichkeit zur Kausativierung gibt es im Deutschen auch Verbpaare wie in (43) und (44), die sich gerade durch das Vorhandensein beziehungsweise Nichtvorhandensein der Argumentstelle für den Auslöser des jeweiligen Prozesses oder Zustands unterscheiden.

Der Baum fiel. - Peter fällte den Baum.

(44) Das Kind erschrickt. - Peter erschreckt das Kind.

Systematische Erweiterungen oder Reduktionen von Argumenten gehen auch häufig mit verbalen Wortbildungprozessen einher. Wie bei den Beispielen der Lügen-Gruppe bereits erwähnt, führt die Kombination eines Verbs mit effiziertem Objekt mit dem umgangssprachlich markierten Präfix "rum-" zu einer Intransitivierung des präfigierten Verbs:

(45) Petra malte ein Bild.

(46) * Petra malte ein Bild rum.

Daß dieser Effekt der Rollenperspektivierung als Konsequenz der durch "rum-" zusätzlich eingebrachten Verlaufsperspektivierung erklärt werden kann, stützt die oben genannte These, daß die verschiedenen Perspektivierungstypen in systematischer Weise aufeinander bezogen werden müssen.

(b) Typ besitzen - gehören: Zwei verbale Ausdrücke unterscheiden sich durch die Art und Weise, in der ihre Argumente syntaktisch angeschlossen werden. Als Beispiel wird oft das Verbpaar "besitzen" und "gehören" genannt, das die Relation zwischen einem Objekt und dem Besitzer dieses Objekts in verschiedener Weise verbalisiert:

(47) Unser Chef besitzt einen Mercedes.

(48) Der Mercedes gehört unserem Chef.

Mit diesen sogenannten lexikalischen Konversen sind gewöhnlich Unterschiede in der Informationsstruktur verbunden: Wird die Rolle des Besitzers thematisch verwendet, wird "besitzen" gewählt; ist das Besitzobjekt Thema, wird man gewöhnlich "gehören" verwenden. Mit bestimmter Fokussierung sind aber die folgenden Sätze durchaus möglich:

(49) Den Mercedes besitzt unser Chef.

(50) Unserem Chef gehört ein Mercedes. 
Zum Typ besitzen-gehören zählen auch die im Zusammenhang mit der Perspektivierung vieldiskutierten Versprachlichungsalternativen für den Situationstyp der kommerziellen Transaktion:

(51) Hans verkaufte Petra einen Sattelschlepper.

(52) Petra kaufte von Hans einen Sattelschlepper.

(53) Petra kaufte Hans einen Sattelschlepper ab.

Mit den Verben "kaufen", "verkaufen" und "abkaufen" können jeweils auf verschiedene Weise die Rollen des Käufers und des Verkäufers realisiert werden. Die in (51) - (53) gezeigten Verbalisierungsalternativen scheinen sich von (47) und (48) nur durch die im Satz nahegelegte Informationsstruktur zu unterscheiden. Daß die Verben "kaufen" und "abkaufen" durchaus über ein unterschiedliches Perspektivierungspotential verfügen, zeigt jedoch der Vergleich von (54) und (55):

(54) Petra kaufte einen Sattelschlepper.

(55) * Petra kaufte einen Sattelschlepper ab.

Während die Rolle des Verkäufers bei "kaufen" fakultativ ist, das heißt, wie in (54) fehlen kann, führt die Nichtverbalisierung derselben Rolle bei "abkaufen" zum grammatisch nicht akzeptablen Satz (55). Die beiden Verben stehen also zusätzlich in einer Opposition vom Typ heiraten-ehelichen (siehe unten).

Auch "kaufen" und "verkaufen" unterscheiden sich in ihrem Perspektivierungspotential, wie die passivischen Konstruktionen in (56) und (57) sichtbar machen:

(56) Ein Sattelschlepper wurde von Hans an Petra verkauft.

(57) * Ein Sattelschlepper wurde von Petra von Hans gekauft.

Zwar sind beide Verben passivierbar, jedoch können in einer Passivkonstruktion wie (57) Käufer und Verkäufer nicht gleichzeitig verbalisiert werden - ein Reflex der formalen Identität der Konstituenten, die beide als "von"-Präpositionalphrase realisiert werden.

Dieses Beispiel sollte jedoch nicht darüber hinwegtäuschen, daß der Perspektivierungszusammenhang zwischen aktivischen und passivischen Konstruktionen tatsächlich dem Typ besitzen-gehören zuzuordnen ist: Dieselben Argumente werden in syntaktisch unterschiedlicher Weise realisiert.

(58) Peter verkaufte den Sattelschlepper.

(59) Der Sattelschlepper wurde von Peter verkauft.

Die Passivierungsmöglichkeiten von Verben können - mit den für natürliche Sprachen typischen 'Ausreißern' - aus deren semantischen und syntaktischen 
Eigenschaften regulär abgeleitet werden. Auch kann in jeder Passivkonstruktion die "von"-Präpositionalphrase (das heißt das Subjekt des Aktivsatzes) weggelassen werden, wodurch das Perspektivierungspotential passivierbarer Verben noch erweitert wird.

Nicht ganz so regulär, aber immerhin doch systematisierbar sind die Perspektivierungsveränderungen, die sich im Bereich der verbalen Wortbildung ergeben. Ein in diesem Zusammenhang vielzitiertes Beispiel ist die "be-"-Präfigierung vom Typ laden-beladen:

(60) Er lädt Heu auf den Wagen.

(61) Er belädt den Wagen mit Heu.

Dieser Perspektivenwechsel zwischen einfachem und präfigiertem Verb wird in Anlehnung an die englische Entsprechung als Lokativalternierung bezeichnet und bildet einen der systematisch beschreibbaren Zusammenhänge zwischen Simplexverben und ihren präfigierten Entsprechungen. Wie bereits erwähnt, ist hier die Veränderung der Rollenperspektivierung meist ein erklärbarer Reflex der mit der Präfigierung vorgenommenen Verlaufsperspektivierung (vergleiche unten 4.2).

(c) Typ heiraten - ehelichen: Zwei verbale Ausdrücke können sich auch darin unterscheiden, ob ihre Argumente in bestimmten Konstruktionen syntaktisch realisiert werden müssen oder nicht. So muß in aktivischen Konstruktionen das Akkusativkomplement von "ehelichen" versprachlicht werden, während das Akkusativkomplement von "heiraten" auch fehlen kann.

(62) Peter heiratet Maria.

(63) Peter heiratet.

(64) * Peter ehelicht.

(65) * Peter und Maria ehelichen.

$\mathrm{Da} \beta$ es sich hierbei nicht um eine konzeptuell bedingte Beschränkung handelt - zum Ehelichen gehören eben zwei! -, zeigt die Nichtakzeptabilität von (65), in dem das koordinierte Subjekt beide Ehepartner bezeichnet.

In valenztheoretischen Verbbeschreibungen werden diese Unterschiede durch das Merkmalspaar obligatorisch-fakultativ erfaßt: Das Akkusativkomplement von "ehelichen" erhielte das Merkmal "obligatorisch", das Akkusativkomplement von "heiraten" das Merkmal "fakultativ". Die mit dieser dichotomen Unterscheidung verbundenen Probleme sollen hier nicht im einzelnen diskutiert werden (vergleiche dazu Blume, 1993; Jacobs, 1994; Storrer, 1996). Wichtig für den hier diskutierten Zusammenhang ist lediglich, daß das Fehlen fakultativer Komplemente in einem Satz in Abhängigkeit vom Äußerungskontext unterschiedlich interpretiert werden kann: 
Bei der sogenannten indefiniten Auslassung fakultativer Komplemente wird zwar die Existenz der dem Komplement entsprechenden Situationsrolle (im Falle "heiraten" also des Ehepartners) postuliert, für den aktuellen Äußerungskontext bleibt ihre Belegung jedoch unspezifiziert. Dieser Fall liegt im folgenden Textbeispiel vor:

Er setzte sich nach Italien ab, ließ das Haar wieder wachsen, heiratete und ging weiter nach Konstantinopel. (Poertner, Die Erben Roms, 1965, S. 85)

Die sogenannte definite Auslassung eines Komplements hingegen ist mit einer Suchanweisung verbunden: Je nach Äußerungskontext kann die Belegung der entsprechenden Rolle aus dem sprachlichen oder situationellen Kontext erschlossen werden, wie im folgenden Textbeispiel, in dem die Belegung der im daß-Satz nicht realisierten Rolle in der unmittelbar folgenden Apposition nachgeliefert wird:

Meine Blechtrommel schloß ich ein und war kaum noch aus dem Zimmer zu bekommen. Dazu kam, daß mein Freund Klepp in jenen Wochen heiratete, ein rothaariges Zigarettenmädchen zu seiner Gattin machte, weil er ihm einmal ein Foto von sich geschenkt hatte. (Grass, Die Blechtrommel, 1964, S. 467)

Während der definiten Auslassung vornehmlich kohärenzstiftende Funktion beim Verstehensprozeß zukommt (vergleiche Schnotz, 1994), indem an bereits Eingeführtes oder Bekanntes angeschlossen werden kann, verändert die indefinite Auslassung tatsächlich die Situationsperspektivierung. Sie ermöglicht es dem Sprecher insbesondere, die Belegung von Situationsrollen unbestimmt zu lassen, sei es, weil sie für den momentanen Aufmerksamkeitsbereich irrelevant ist oder weil der Sprecher die Belegung zum Zeitpunkt der Äußerung selbst nicht kennt; sei es, weil eine Spezifizierung dieser Rolle seiner momentanen Interessenslage entgegensteht (für Beispiele vergleiche Heringer, 1984; Storrer, 1992, S. 267-274).

Oppositionen vom Typ heiraten-ehelichen werden systematisch durch bestimmte sprachliche Mittel induziert. Es wurde mit (52) versus (54) bereits gezeigt, daß die in aktivischen Sätzen obligatorischen Subjektstellen in einer Passivkonstruktion als grundsätzlich fakultative "von"-Präpositionalphrasen erscheinen. Insofern unterscheiden sich Funktionsverbgefüge mit passivischer Funktion auch von den entsprechenden Passivkonstruktionen:

(66) Das Programm wird (von Herrn Schlaule) eingesetzt.

(67) Das Programm kommt zum Einsatz.

Während in der Passivkonstruktion in (66) die Agens-Rolle (Herr Schlaule) als fakultative "von"-Präpositionalphrase angeschlossen werden kann, ist die Reali- 
sierung dieser Rolle in der passivischen Funktionsverb-Konstruktion (67) nicht mehr möglich.

\subsection{Verlaufsperspektivierung im Deutschen}

Eine Art von Verlaufsperspektivierung wird traditionell unter den grammatischen Kategorien des Aspekts oder der Aktionsart abgehandelt: Ein im Situationstyp standardmäßig erfaßter Zustand oder Prozeß wird im Hinblick auf seine Entstehung, seinen Fortbestand oder seine Beendigung perspektiviert. Als Mittel dienen hierzu einerseits Verbalpräfixe und -partikeln:

(68) Die Rose blüht.

(69) Die Rose erblüht. (inchoativ)

(70) Die Rose verblüht. (terminativ)

Es existiert andererseits eine Reihe von Funktionsverben, die eine inchoative und durative Verlaufsperspektivierung induzieren:

(71) Der Zug bewegt sich.

(72) Der Zug kommt in Bewegung. (inchoativ)

(73) Der Zug bleibt in Bewegung. (durativ)

Wie am Beispiel der kausativierenden Funktionsverben "bringen" und "versetzen" in (40) und (42) gezeigt, kann die Veränderung der Verlaufsperspektive auch mit systematischen Konsequenzen für die Möglichkeiten der Rollenperspektivierung verbunden sein. Ein noch deutlicherer Zusammenhang zwischen Verlaufs- und Rollenperspektivierung besteht zwischen Simplexverben und ihren präfigierten Varianten. Insbesondere verbale Präfixe wie "an-", "auf-", "durch-", "rum-" etc. kombinieren auf festgelegte Weise Teile ihrer präpositionalen Bedeutung mit der Bedeutung des Simplexverbs. Die Veränderungen der Rollenperspektive können dann als direkte Konsequenz der durch das Präfix eingebrachten Verlaufsperspektive erklärt werden. In Abschnitt 3.2 wurde dieser Zusammenhang am Beispiel der Lügen-Gruppe ("zusammenlügen", "sich durchlügen" und "rumlügen") bereits erwähnt; detaillierte Analysen finden sich in Stiebels (1994).

Die sprachlichen Mittel zur Rollen- und Verlaufsperspektivierung sind hiermit sicher nicht erschöpfend beschrieben. Die gewählten Beispiele zeigen jedoch deutlich, in welch systematischer Weise dem Sprecher Alternativen zur Wahl stehen, um eine situationsadäquate Auswahl an Situationsrollen versprachlichen zu können. Diese Alternativen können genutzt werden, um bei einer Situationsbeschreibung bestimmte Aspekte im dunkeln zu lassen beziehungsweise den Adressaten nicht mit Unerheblichem zu belästigen, wobei na- 
türlich nicht jeder real existierende Sprecher aktiv über das gesamte Spektrum an Möglichkeiten verfügen wird.

\section{Diskussion}

Der hier vorgeschlagene Ansatz relationiert Argumentstellen verbaler Ausdrücke auf der sprachlichen Beschreibungsebene mit Situationsrollen und verlaufsbezogenen Parametern auf der konzeptuellen Beschreibungsebene. Die Grundeinheiten der konzeptuellen Beschreibungsebene sind Situationstypen, verstanden als kognitive Schemata, unter die individuelle Situationen subsumiert werden können. Speziellere Situationstypen können mit allgemeineren Situationstypen in Beziehung gesetzt werden und von diesen Merkmale und Situationsrollen vererbt bekommen. Die Situationsrollen sind durch Merkmale charakterisiert, die in ganz allgemeiner Weise festlegen, durch welche Art von Entitäten sie belegt werden können. So muß trivialerweise der Sprecher im Situationstyp "Kommunikative Handlung" zum sprachlichen Ausdruck und zum intentionalen Handeln befähigt sein; diese Mindestanforderung gilt dann auch für entsprechende Situationsrollen speziellerer Situationstypen, zum Beispiel dem Situationstyp "Lüge". Pro Situationstyp wird dann die Menge der verbalen Ausdrücke bestimmt, mit denen er in der betreffenden Einzelsprache beschrieben werden kann.

Auf der sprachlichen Beschreibungsebene werden diejenigen verbalen Ausdrücke einer Einzelsprache, die sich zur Bezeichnung der allgemeineren und spezielleren Situationstypen eignen, zu Mengen und Untermengen zusammengefaßt, die den Synsets im relationalen Ansatz von WordNet vergleichbar sind (vergleiche Fellbaum in diesem Band). Die zu einer Menge beziehungsweise Untermenge gehörigen verbalen Ausdrücke haben zwar dieselbe extensionale Bedeutung (sie denotieren dieselbe Klasse von Situationen), beschreiben diese jedoch zumeist auf unterschiedliche Weise, sind also intensionsverschieden. Für den vorliegenden Ansatz sind hierbei insbesondere die Unterschiede im Potential zur Rollen- und Verlaufsperspektivierung relevant, die auf der sprachlichen Beschreibungsebene auf folgende Weise erfaßt werden: Die Möglichkeiten der Rollenperspektivierung sind durch Abbildungen von verbspezifischen Valenzstellen auf Situationsrollen festgelegt. Eine solche Zuordnung wurde in der in Abschnitt 3 dargestellten Tabelle 1 exemplifiziert, in der Situationsrollen für den Situationstyp "Lüge" auf einen Teil der bezeichnungsgeeigneten Verben abgebildet werden. Allerdings erfaßt diese einfache Zuordnung bislang weder verlaufsspezifische Parameter, noch sind die in Abschnitt 3 diskutierten sprachlichen Mittel zur Perspektivenveränderung systematisch erfaßt. Diese einfache Repräsentation muß durch um Merkmale angereicherte Lexikoneinträge verfeinert werden. Solche Lexikoneinträge enthalten Verbargumente, die dann mit den Situationsrollen der ebenfalls als Merkmalstrukturen repräsentierten Situationstypbeschreibungen koindiziert werden können. Über diese Merkmalstruk- 
turen können dann Prozesse wie Passivierung und Rezessivierung und deren Auswirkungen auf das Perspektivierungspotential generell formuliert werden. Lexikalisierte Wortbildungsprodukte können als atomare verbale Einheiten mit spezifischem Perspektivierungspotential behandelt werden; für produktive Wortbildungsmuster mit systematischem Einfluß auf die Rollen- und Verlaufsperspektivierung muß nach anderen Lösungen gesucht werden. Da eine Zerlegung von Verbbedeutungen in semantische Primitive in diesem Ansatz vermieden werden soll, müssen produktive Derivationsmorpheme als Grundeinheiten in die Lexikonbeschreibung mit aufgenommen werden, die in spezifischer Weise das Perspektivierungspotential der mit ihnen kombinierten Verbstämmme modifizieren.

Durch die Relationierung von Mengen sprachlicher Ausdrücke mit Situationstypen unterscheidet sich der hier vorgeschlagene Ansatz von Modellen, in denen unterschiedliche syntaktische Konstruktionen auf jeweils unterschiedliche konzeptuelle Strukturen abgebildet werden (zum Beispiel Jackendoff, 1991). Eine derartige Abbildung macht die Ausgestaltung der als sprachunabhängig postulierten konzeptuellen Strukturebene in hohem Maße einzelsprachabhängig und führt zudem zu einer inflationären Anzahl polysemer Lexikoneinträge. Die aus dem letztgenannten Problem resultierende Aufblähung des Lexikons durch polyseme Einträge wird im Paradigma der Zwei-Ebenen-Semantik (zum Beispiel Lang, 1992) durch die Differenzierung zwischen einer semantischen und einer konzeptuellen Repräsentationsebene vermieden: Auf der semantischen Ebene werden die invarianten Bedeutungsbestandteile sprachlicher Ausdrücke festgehalten; die Fixierung der aktuellen Äußerungsbedeutung erfolgt dann durch eine vom Kontext gesteuerte konzeptuelle Interpretation. Über die Ausgestaltung der konzeptuellen Ebene und die Interaktion zwischen semantischer und konzeptueller Ebene besteht, wie der kritische Vergleich von Meyer (1994) zeigt, jedoch auch innerhalb des Zwei-Ebenen-Paradigmas noch kein Konsens. Die von Meyer verglichenen Arbeiten behandeln zudem meist die Analyserichtung, das heißt den Einfluß von kontextuellen Parametern auf die Interpretation sprachlicher Ausdrücke. Im Gegensatz dazu zielt das vorliegende Modell auf die Sprachgenerierungsrichtung ab, indem es das Zusammenspiel von außersprachlich-situationsspezifischen, kognitiv situationstyp-bezogenen und sprachlichen Parametern beschreibt. Auf dieser Grundlage läßt sich im Hinblick auf eine individuelle Äußerungssituation entscheiden, welche verbalen Ausdrücke in welcher Konstruktion zur Verbalisierung einer bereits konzeptualisierten Situationsbeschreibung geeignet sind.

Ein wichtiger Unterschied zwischen dem hier vorgeschlagenen Beschreibungsansatz und anderen Modellen besteht darin, daß die semantische Beziehung zwischen Verbbedeutung und Verbargumenten nicht mit Hilfe semantischer Rollen (Agens, Patiens etc.) spezifiziert wird. Sie ergibt sich vielmehr durch die Zuordnung von Argumenten zu Situationsrollen: Daß Peter in einem Satz wie (16) oder (19) beim Hörer willentlich eine Fehlinformation weitergibt, $\mathrm{da} ß$ er also als prototypischer Agens auftritt, ist durch den Situationstyp vorge- 
geben, das heißt durch das Wissen darüber, welche Rolle der Lügner bei einer kommunikativen Handlung vom Typ "Lüge" einnimmt. Das Problem der Definition von Anzahl und Status semantischer Rollen (vergleiche Dowty, 1991) ist bei diesem Ansatz umgangen. Es verlagert sich allerdings auf die konzeptuelle Ebene, auf der die Anzahl der Situationsrollen für einen gegebenen Situationstyp festgelegt und Situationsrollen verschiedener Situationstypbeschreibungen miteinander verbunden werden müssen, zum Beispiel die Rolle des Sprechers im allgemeinen Situationstyp "Kommunikative Handlung" mit der Rolle im spezifischeren Situationstyp "Lüge".

Aufgrund seiner Ausrichtung auf die Generierung eignet sich das Modell als theoretische Grundlage für lexikographische Beschreibungen, die auf die Sprachproduktion ausgerichtet sind. Verbbeschreibungen in deutschen ein- und mehrsprachigen Wörterbüchern sind in Produktionssituationen nämlich nur in sehr begrenztem Maße hilfreich (Wiegand, 1985), da sie sich meist mit der Unterscheidung von transitiven und intransitiven Verben begnügen, einer Einteilung, die weder die syntaktische Kategorisierung noch semantische Belegungsregeln für die Verbargumente berücksichtigt.

Dies gilt für alphabetische Wörterbücher genauso wie für Wörterbücher, in denen der Wortschatz nach onomasiologischen Prinzipien angeordnet wurde. Auf der Grundlage des vorliegenden Modells könnte das Zusammenspiel von außersprachlich-situationsspezifischen, situationstyp-bezogenen und sprachlichstrukturellen Parametern in neuartiger Weise lexikographisch erfaßt und beschrieben werden. In Sprachproduktionssituationen können solche Beschreibungen dann zur Wahl eines geeigneten verbalen Ausdrucks herangezogen werden. Außerdem können die entstehenden Sprachbeschreibungen sowohl von muttersprachlichen Sprechern (wie im vorherigen Abschnitt erwähnt, stehen natürlich nicht jedem Sprecher einer Einzelsprache alle sprachlichen Möglichkeiten aktiv zur Verfügung) als auch von Lehrern und Lernern des Deutschen als Fremdsprache zur Wortschatzerweiterung genutzt werden.

Er war bislang jedoch äußerst schwierig, derartig komplexe Bezüge zwischen Verbbeschreibungen und Situationstypbeschreibungen in einem Wörterbuch darzustellen. Dies zeigt sich an lexikographischen Spezialwörterbüchern mit vergleichbarer Zielsetzung, wie bei dem Valenzwörterbuch Verben in Feldern (Projektgruppe Verbvalenz, 1981; Schumacher, 1986) oder bei dem große Teile des deutschen Verbwortschatzes erfassenden Verbwörterbuch Deutsche Verben (Ballmer \& Brennenstuhl, 1981). Aktuelle Projekte zur onomasiologischen Erfassung des Wortschatzes wie das WordNet-Projekt (Fellbaum in diesem Band) oder das in Abschnitt 3 skizzierte ESKA-Projekt verwalten ihre Beschreibungen deshalb mit Computerprogrammen, die dem Benutzer vielfältigen und flexiblen Zugriff auf die Daten ermöglichen. Künftig kann auch die Hypermedia-Technologie genutzt werden, um textuelle Beschreibungen mit Graphik, Ton, Animation und Videosequenzen anzureichern; hierdurch könnten Situationstypbeschreibungen und Perspektivierungsalternativen nicht nur abstraktsymbolisch, sondern auch graphisch repräsentiert und durch Videosequenzen 
exemplifiziert werden. Der lexikographischen Umsetzung einer situationstypbezogenen Verbbeschreibung werden damit völlig neue Möglichkeiten eröffnet.

\section{Literatur}

Ballmer, T. \& Brennenstuhl, W. (1986). Deutsche Verben. Eine sprachanalytische Untersuchung des deutschen Verbwortschatzes. Tübingen: Narr.

Blume, K. (1993). Valenz deutscher Verben und (Nicht-) Notwendigkeit (Arbeiten des Sonderforschungsbereichs 282 "Theorie des Lexikons"). Düsseldorf: Heinrich Heine Universität.

Dowty, D. (1991). Thematic proto-roles and argument selection. Language, 67, 547-619.

Fellbaum, Ch. (in diesem Band). WordNet: Ein semantisches Netz als Bedeutungstheorie (S. 211-230).

Fillmore, C. J. (1977). Scenes-and-frames-semantics. In A. Zampolli (Hrsg.), Linguistic structures processing (S. 55-81). Amsterdam: North-Holland.

Grass, G. (1964). Die Blechtrommel. Frankfurt/M.: Fischer.

Grice, H. P. (1975). Logic and conversation. In P. Cole \& J. Morgan (Hrsg.), Syntax and semantics. Vol. 3: Speech acts (S. 41-58). New York: Cambridge Harvard University Press.

Harras, G. (1995). Eine Möglichkeit der kontrastiven Analyse von Kommunikationsverben. In H. P. Kromann \& A. L. Kjær (Hrsg.), Von der Allgegenwart der Lexikologie. Kontrastive Lexikologie als Vorstufe zur zweisprachigen Lexikographie (Akten des internationalen Werkstattgesprächs zur kontrastiven Lexikologie 29.-30. 10. 1994 in Kopenhagen) (S. 102-113). Tübingen: Niemeyer.

Harras, G. \& Winkler, E. (1994). A model for describing speech act verbs. The semantic base of a polyfunctional dictionary. In W. Martin, W. Meijs, M. Moerland, E. ten Pas, P. van Sterkenburg \& P. Vossen (Hrsg.), Euralex 1994. Proceedings (Papers to the 6th EURALEX International Congress on Lexicography in Amsterdam) (S. 440 - 449). Amsterdam.

Heringer, H. J. (1984). Neues von der Verbszene. In G. Stickel (Hrsg.), Pragmatik in der Grammatik (Jahrbuch 1983 des Instituts für deutsche Sprache) (S. 34-64). Düsseldorf: Schwann.

Jackendoff, R. S. (1991). Semantic structures. Cambridge, MA: MIT Press.

Jacobs, J. (1994). Das lexikalische Fundament der Unterscheidung von obligatorischen und fakultativen Ergänzungen. Zeitschrift für Germanistische Linguistik, 22, 284-319.

Lang, E. (1992). Semantische vs. konzeptuelle Struktur: Unterscheidung und Überschneidung. In M. Schwarz (Hrsg.), Kognitive Semantik/Cognitive Semantics (Tübinger Beiträge zur Linguistik 395) (S. 25-40). Tübingen: Narr.

Meyer, R. (1994). Probleme von Zwei-Ebenen-Semantiken. Kognitionswissenschaft, 4, 32 - 46.

Minsky, M. (1980). A framework for representing knowledge. In D. Metzing (Hrsg.), Frame conceptions and text understanding (S. 1-26). Berlin: de Gruyter.

Mudersbach, K. (1984). Kommunikation über Glaubensinhalte - Grundlagen der epistemistischen Linguistik. Berlin: de Gruyter.

Poertner, F. (1965). Die Erben Roms. Düsseldorf: Econ.

Polenz, P. von (1987). Funktionsverben, Funktionsverbgefüge und Verwandtes. Vorschläge zur satzsemantischen Lexikographie. Zeitschrift für Germanistische Linguistik, 15, 169-189.

Projektgruppe Verbvalenz (1981). Konzeption eines Wörterbuchs deutscher Verben (Forschungsberichte des Instituts für deutsche Sprache, 45). Tübingen: Narr.

Reimer, U. (1987). FRM: Ein Frame-Repräsentationsmodell und seine formale Semantik - Zur Integration von Datenbank- und Wissensrepräsentationsansätzen. Heidelberg: Springer.

Schank, R. C. \& Abelson, R. P. (1977). Scripts, plans, goals and understanding. An inquiry into human knowledge structures. Hillsdale, NJ: Erlbaum. 
Schnotz, W. (1994). Aufbau von Wissensstrukturen. Untersuchungen zur Kohärenzbildung beim Wissenserwerb mit Texten (Fortschritte der psychologischen Forschung 20). Weinheim: Psychologie Verlags Union.

Schumacher, H. (Hrsg.). (1986). Verben in Feldern. Valenzwörterbuch zur Syntax und Semantik deutscher Verben. Berlin: de Gruyter.

Stiebels, B. (1994). Lexikalische Argumente und Adjunkte. Zum sematischen Beitrag von verbalen Präfixen und Partikeln. Unveröffentl. Dissertation. Düsseldorf: Heinrich-Heine-Universität.

Storrer, A. (1992). Verbvalenz. Theoretische und methodische Grundlagen ihrer Beschreibung in Grammatikographie und Lexikographie. Tübingen: Niemeyer.

Storrer, A. (1996). Wie notwendig sind obligatorische Valenzstellen? - Faktoren der Weglaßbarkeit von Valenzstellen im Text. In G. Gréciano \& H. Schumacher (Hrsg.), Syntaxe structurale et opérations mentales. Akten des deutsch-französischen Kolloquiums anläßlich der 100. Wiederkehr seines Geburtstages 1993 (S. 225-238). Tübingen: Niemeyer.

Storrer, A. \& Schwall, U. (1995). Description and acquisition of multiword lexemes. In P. Steffens (Hrsg.), Machine translation and the lexicon. Third International EAMT Workshop Heidelberg, Germany, April 26-28, 1993, Proceedings (Lecture Notes in Artificial Intelligence 898) (S. 35-51). Heidelberg: Springer.

Wiegand, H. E. (1985). Fragen zur Grammatik in Wörterbuchbenutzungsprotokollen. Ein Beitrag zur empirischen Erforschung der Benutzung einsprachiger Wörterbücher. In H. Bergenholtz \& J. Mugdan (Hrsg.), Lexikographie und Semantik (Akten des Essener Kolloquiums zur Grammatik im Wörterbuch 28. - 30.6.1984) (S. 20-98). Tübingen: Niemeyer.

Winkler, E. (in diesem Band). Kommunikationskonzepte und Kommunikationsverben (S. 256-276). 